\title{
LTX-315: a first-in-class oncolytic peptide that reprograms the tumor microenvironment
}

The oncolytic peptide LTX-315, which has been de novo designed based on structureactivity relationship studies of host defense peptides, has the ability to kill human cancer cells and induce specific anticancer immune response when injected locally into tumors established in immunocompetent mice. The oncolytic effect of LTX-315 involves perturbation of plasma membrane and the mitochondria with subsequent release of danger-associated molecular pattern molecules, which highlights the ability of LTX315 to induce complete regression and protective immune responses. Treatment with LTX-315 reprograms the tumor microenvironment by decreasing the local abundance of immunosuppressive cells and by increasing the frequency of effector T cells.

First draft submitted: 28 March 2017; Accepted for publication: 20 April 2017; Published online: 8 May 2017

Keywords: cancer $\bullet$ DAMP $\bullet$ immunotherapy $\bullet$ oncolytic peptide

Host defense peptides, often also denoted antimicrobial peptides or cationic antimicrobial peptides (CAPs) since most of them are abundant in cationic residues, constituting an important part of the innate immune system and are present in virtually all species of life [1-3]. To date, more than 2800 antimicrobial peptides isolated from species ranging from bacteria, fungi, plants to mammals have been reported and are curated in the Antimicrobial Peptide Database [4]. Their most studied function is the defense against bacteria and, consequently, a number of naturally occurring and synthetic analogues have been evaluated in clinical trials as future antibiotics [5]. The antimicrobial activity has in many cases been ascribed to peptide-membrane interactions, in which negatively charged constituents on bacterial surfaces have been implied as the initial targets for interaction with cationic side chains on CAPs. CAPs have also been found to cross the bacterial membrane to act on intracellular targets [6]. It is not uncommon for CAPs to also demonstrate cytotoxic activity against cancer cells at concentrations that are not toxic to normal cells $[7,8]$, which can be explained by a higher abundance of anionic membrane components on cancer cells compared with normal cells $[7,8]$. Thus, CAPs may display a level of selectivity not commonly displayed by chemotherapeutic drugs.

Several different models describing the peptide-lipid interactions leading to membrane disruption by CAPs have been suggested [9-15], and the ability to adopt amphipathic structures with separated cationic (Lys and Arg residues) and hydrophobic regions (Val, Leu, Ile, Phe and Trp residues) has been established as important [16,17]. Furthermore, some of the CAPs demonstrate immunomodulatory activities such as chemotaxis and an induction of cytokine release (see [18] for a recent review).

Bovine lactoferricin (LfcinB) [19] is a well-studied 25 amino acid CAP that displays selective antimicrobial and anticancer properties [20-22]. This peptide is derived from the pepsin-mediated hydrolysis of the iron-binding bovine glycoprotein lactofer-
Baldur Sveinbjørnsson ${ }^{* 1,2,}$, Ketil Andre Camilio ${ }^{2,3}$, Bengt Erik Haug $^{4}$ \& Øystein Rekdal ${ }^{1,2}$ ${ }^{1}$ Department of Medical Biology, Faculty of Health Sciences, UiT The Arctic University of Norway, NO-9037 Tromsø, Norway

Lytix Biopharma, Gaustadalléen 21, NO-0349 Oslo, Norway

${ }^{3}$ Department of Tumor Biology, Institute for Cancer Research, Oslo University Hospital, NO-0379 Oslo Norway ${ }^{4}$ Department of Chemistry \& Centre for Pharmacy, University of Bergen, Allégaten 41, NO-5007 Bergen, Norway *Author for correspondence: baldur.sveinbjornsson@lytixbiopharma.com 
rin $[19,23]$, and is present in exocrine secretions such as milk, saliva and in the secretory granules of neutrophils [20]. LfcinB contains residues 17-41 of the parent lactoferrin protein, and has a cyclic structure due to a disulphide bond between two Cys residues. In the bovine lactoferrin protein, residues 17-28 comprise a helical segment [24], whereas the 25-mer peptide adopts a twisted antiparallel $\beta$-sheet structure in solution [25]. Noteworthily, both structures are highly amphipathic. While the linear version of LfcinB proved to be less active than the cyclic parent peptide [21], linear analogs with a high cytotoxic activity could be obtained by focusing on the helical part of the parent protein. Through these studies, it has further been confirmed that an amphipathic secondary structure as well as the nature, size and positioning of aromatic amino acids is of importance for activity [26-28].

The vast amount of SAR-data on lactoferricinderived, and also synthetic model peptides has facilitated the de novo design of 9-mer cationic peptides in which counterproductive residues have been deleted and the properties of residues identified as important for anticancer activity have been optimized, thereby resulting in a series of 9-mers composed of five cationic residues, two or three tryptophan residues and one or two bulky and lipophilic unnatural residues [29]. These peptides were found to be highly effective against both drug-resistant and drug-sensitive cancer cells, and displayed a lower activity toward normal cells. One of the candidates developed, named 'LTX-315' (Figure 1) displayed selective anticancer activity in preclinical studies.

LTX-315 has been shown to be highly active against a wide variety of both murine and human cancer cell lines in vitro [29-34].

\section{Discussion}

In recent years, immunogenic cell death (ICD) has emerged as a new concept in cancer therapy [35]. ICD is initiated by the release of so-called danger-associated molecular pattern molecules (DAMPs) such as calreticulin, ATP and high mobility group box protein 1 (HMGB1), and has the potential of stimulating an immune response against antigens derived from dying cancer cells [35]. Further, only cancer therapies capable of inducing ICD can instigate a tumor-specific antitumor response through activation of dendritic cells and consequent activation of specific T-cell activity.

Local immunotherapy of cancer capable of mediating abscopal effects, and hence long-term protection from metastatic relapse, holds great potential for therapeutic intervention in oncology [36]. By reprogramming the tumor microenvironment through the eradication of local immune suppressive cells such as regulatory $\mathrm{T}$ cells (Tregs) followed by the infiltration of cancer killing $\mathrm{CD}^{+}$cytotoxic $\mathrm{T}$ cells (CTLs), one may reinstate systemic anticancer immune responses, hence eliminating all deposits of neoplastic cells. The intratumoral treatment of solid tumors with LTX-315 has resulted in growth inhibition, complete regression and long-lasting tumor-specific immune responses in a variety of different experimental animal models. Typically, tumors ranging from 60 to $100 \mathrm{~mm}^{3}$ were injected with $20 \mathrm{mg} / \mathrm{ml}$ LTX-315 at a volume of $50 \mu \mathrm{l}$ (1 mg/injection) once per day for three consecutive days without any sign of systemic adverse events observed.

LTX-315 induces ICD through its membranolytic mode of action, leading to the release of potent immunostimulants in addition to a wide spectrum of tumor antigens, thus creating an essential premise for tumor-specific immune responses.

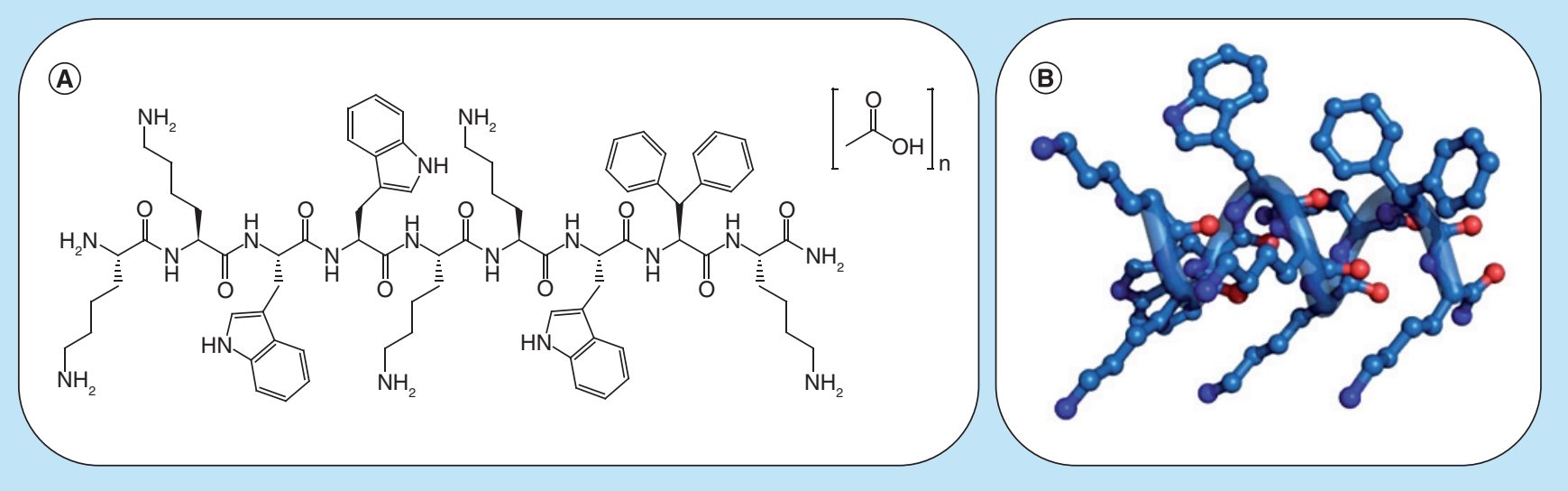

Figure 1. Structure of LTX-315. (A) Amino acid sequence of LTX-315 (K-K-W-W-K-K-Dip-K-NH ( $^{2}$; (B) 3D representation of an idealized helical conformation of LTX-315.

Generated in Pymol (courtesy of Ulli Rothweiller, UiT). 


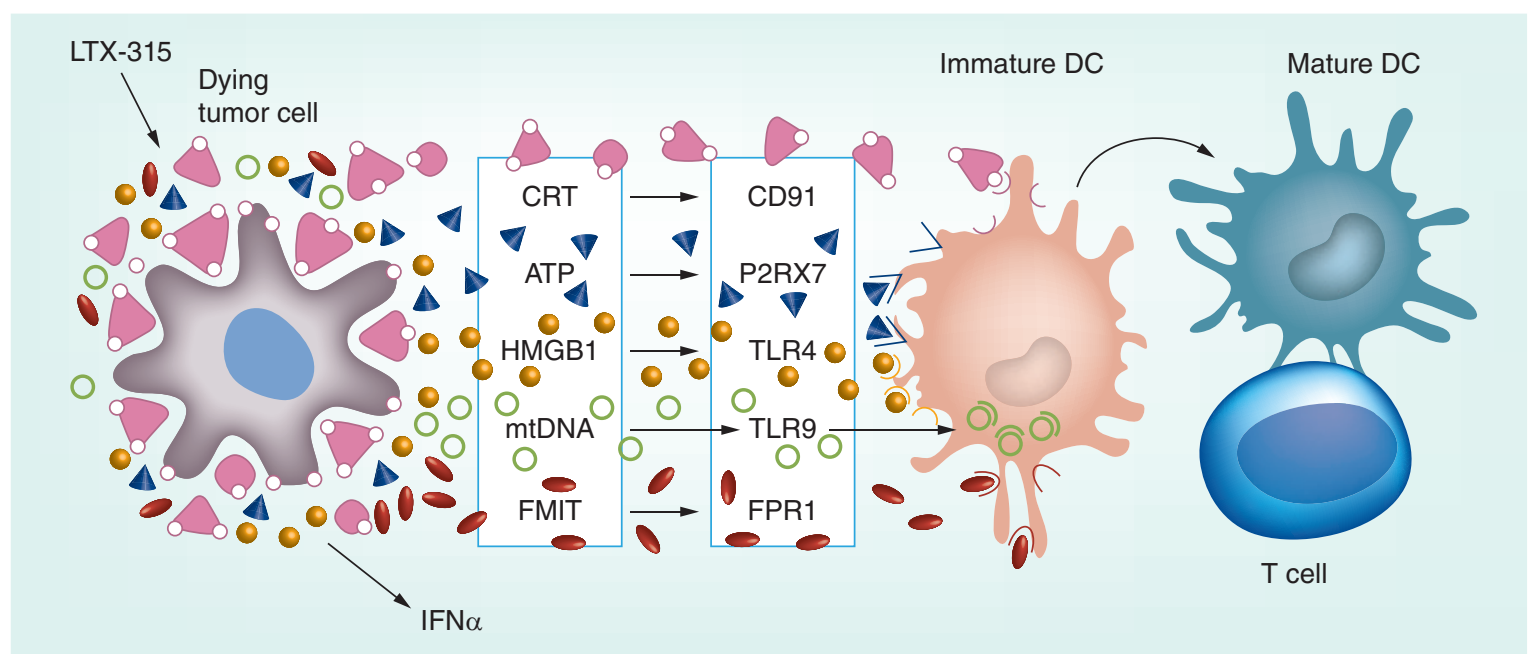

Figure 2. LTX-315 induces immunogenic cell death in cancer cells. When treated with LTX-315, dying cancer cells release DAMPs such as calreticulin, ATP, HMGB1, mtDNA and formyl peptides. DAMPs bind to specific receptors on antigen-presenting cells such as dendritic cells and promotes their maturation and engulfment of tumor-antigens with subsequent presentation to $T$ cells and execution of effective immune response.

DAMP: Damage-associated molecular pattern; FMIT: Mitochondrial N-formyl peptide; mtDNA: Mitochondriaderived DNA.

Intratumoral treatment with LTX-315 resulted in a complete regression of orthotopic B16 melanomas in $80 \%$ of animals. LTX-315-treated tumors harvested for immunohistochemistry displayed a significant increase in tumor-infiltrating $\mathrm{T}$ cells (TILs) and a change in TIL distribution in the tumor microenvironment, going from a peritumoral location to a more intratumoral location over time. Moreover, when previously cured animals were rechallenged with viable tumor cells they demonstrated a systemic protection against tumor formation, both orthotopically in the skin and in a lung metastasis model in which the tumor cells were injected intravenously 9 months post-treatment [31].

The treatment of tumors with LTX-315 leads to a disintegration of the plasma membrane and cytoplasmic organelles with a subsequent release of DAMPs such as ATP, calreticulin, HMGB1 and cytochrome $c$, (Figure 2) in addition to tumor antigens, including neoantigens $[32,33,37,38]$. This results in an increase in TILs, TIL clonality and the number of clones with greater abundance in the tumor microenvironment. The treatment with LTX-315 also reprograms the tumor microenvironment by decreasing the local abundance of immunosuppressive Tregs and myeloid-derived suppressor cells [37].

Furthermore, an abscopal effect on nontreated lesions has been observed. When mesenchymal subcutaneous sarcomas in PVG rats (rTMSCs) were injected with LTX-315, all animals treated went into complete remission. More remarkably, when only one out of three lesions in a three-tumor model mimicking tumor cell dissemination and metastasis was treated with LTX-315, both the treated lesion and the disseminated tumors were eradicated. Nontreated lesions were heavily infiltrated with $\mathrm{CD}^{+}$ and $\mathrm{CD} 8^{+} \mathrm{T}$ cells, consequently indicating a strong and immediate systemic effect following the LTX315-based therapy [Nestvold J, Wang M-Y, Camilio K ET AL. OnColytic peptide LTX-315 induces an immune-mediated ABSCOPAL EFFECT IN A RAT SARCOMA MODEL; MANUSCRIPT IN PREPAration].

Long-term protective immune responses were further demonstrated as previously cured animals were protected against s.c. and i.p. rechallenge 14 months after treatment. In addition, adoptive transfer of splenocytes allowed for transfer of the protective immunity of cured animals to irradiated naive recipients. Moreover, the transferred protective immunity was tumor-specific, preventing the tumor formation of rTMSCs though not Roser's T-cell leukemia after s.c. inoculation [Nestrold J, Wang M-Y, Camilio K et al. OnCOlytic PEPtide LTX-315 induces AN IMMUNE-MEdiated ABSCOPAL EFFECT IN A RAT SARCOMA MODEL; MANUSCRIPT IN PREPARATION].

Emerging data indicate a correlation between pre-existing T-cell content in the tumortumor microenvironment, in particular the cytotoxic $\mathrm{CD}^{+} \mathrm{T}$ cells, and clinical response. Thus, patients with tumors who lack immune T-cell infiltration, so-called 'cold' tumors, require certain therapeutic interventions to restore T-cell entry, which enables responsiveness to immunotherapy [39]. 
The ability of LTX-315 to increase T-cell infiltration, clonality and the diversity of $\mathrm{T}$ cells indicates that LTX-315 has a potential to reshape the tumor microenvironment, which makes LTX-315 potentially ideal as a combination partner for other immunotherapies, including immune checkpoint inhibitors (anti-CTLA4/anti-PD-1).

Indeed, in some preclinical tumor models, a combination of LTX-315 and immune checkpoint inhibitors (anti-CTLA4) demonstrates a significant synergy. Yamazaki et al [37] showed that LTX-315 acted as an immunogenic cytotoxic compound in vivo when used locally against MCA205 sarcomas. Small MCA205 tumors $\left(20-25 \mathrm{~mm}^{2}\right)$ went into complete regression when treated intratumorally with LTX315. The LTX-315-mediated anticancer effects were shown to be $\mathrm{CD}^{+}$- and $\mathrm{CD} 8^{+}$-T-cell-dependent as depletion experiments demonstrated that the removal of these cells abrogated the antitumor effects. In addition, treatment with LTX-315 increased the intratumoral ratio of CTLs over Tregs. Furthermore, when treating larger MCA205 sarcomas $\left(>40 \mathrm{~mm}^{2}\right)$ with LTX-315 in combination with CTLA4 mAb, a significant synergistic effect was observed. Peptide therapy demonstrated that membrane CTLA4 molecules were clearly upregulated by the peptide. An initial treatment with CTLA4 blockade followed by LTX-315 exhibited a major synergistic interaction, compared with either monotherapy. When both therapies were injected locally, Yamazaki and coworkers obtained a strong therapeutic synergy, both on ipsilateral and contralateral MCA205 sarcomas. This demonstrates that LTX-315 is capable of markedly enhancing the immune-mediated anticancer effects induced by CTLA4 blockade, even in tumors that were primarily resistant to CTLA4 $\mathrm{mAb}$ [37].

Other potential combination therapies or applications will become of high interest as well, for example combinations with adoptive T-cell therapies, CAR-T cell-therapy etc. These therapies are dependent on the isolation and extraction of high-quality $\mathrm{T}$ cells (from 'hot' tumors) in order to produce tumorspecific $T$ cells of a proper quality that can be reintroduced back to the patient. This so-called 'T-cell primer' application would further increase the potential use of LTX-315. Finally, in preclinical models, a combination of LTX-315 and chemotherapeutic agents, such as cyclophosphamide and doxorubicin exhibits significant synergy compared with either monotherapy [40].

The primary objective of local oncolytic therapies is to induce immunogenic cancer cell death in order to stimulate a tumor-specific immune response against residual cancer cells. The unique membranolytic mode of action of LTX-315 leads to the release of danger signals or damage-associated molecular pattern molecules and tumor antigens. These potent immunostimulants prompt the recruitment and activation of professional antigens presenting cells such as dendritic cells which, in turn, can process and present the released tumor antigens and neoantigens to $\mathrm{T}$ cells. The subsequent T-cell activation and tumor-specific immune responses are characteristics essential for an abscopal effect against nontreated distant lesions and long-lasting immune protection against metastatic relapse.

In addition to a modest and predictable safety profile, promising Phase I clinical data has demonstrated to date that LTX-315 induces a partial and complete regression in injected tumors, durable stable disease (global tumor response by CT scan) and significant increases in TILs, indicating a systemic immune response in some patients [41].

A Phase Ib study combining LTX-315 with ipilimumab (anti-CTLA4) in malignant melanoma patients, as well as LTX-315 with pembrolizumab (anti-PD-1) in metastatic breast cancer patients is ongoing.

\section{Future perspective}

LTX-315 is a first-in-class oncolytic peptide for intratumoral injection. Previous data have shown that LTX-315 has the potential to stimulate anticancer immune responses in experimental preclinical animal models. Local treatment of tumor results in increased T-cell infiltration and anticancer immune responses that also suppress growth of distant tumors (abscopal effect). Thus, LTX-315 is ideal as a combination partner for other immunotherapies, including immune checkpoint inhibitors (anti-CTLA4/ anti-PD-1).

\section{Financial \& competing interests disclosure}

B Sveinbjørnsson, KA Camilio, BE Haug and $\varnothing$ Rekdal are shareholders in Lytix Biopharma. B Sveinbjørnsson and Ø Rekdal are employed by Lytix Biopharma. The authors have no other relevant affiliations or financial involvement with any organization or entity with a financial interest in or financial conflict with the subject matter or materials discussed in the manuscript apart from those disclosed.

No writing assistance was utilized in the production of this manuscript.

\section{Open access}

This work is licensed under the Attribution-NonCommercialNoDerivatives 4.0 Unported License. To view a copy of this license, visit http://creativecommons.org/licenses/by-nc-nd/4.0/ 
Executive summary

- Oncolytic 9-mer cationic peptides were designed based on SAR-studies on the cationic antimicrobial peptide bovine lactoferricin

- One candidate, LTX-315 was shown to be highly effective against a broad panel of both drug-resistant and drug-sensitive cancer cells with lower activity against normal cells.

- LTX-315 induces immunogenic cell death as defined through the release of danger-associated molecular patterns from tumor cells with subsequent activation of specific antitumor immune responses.

- Intratumoral administration of LTX-315 in animal models results in tumor necrosis and the infiltration of immune cells into the tumor tissue followed by complete remission of tumor.

- Treatment with LTX-315 reshapes the tumor microenvironment by decreasing the local abundance of immunosuppressive cells and increasing the frequency of effector T cells.

- The ability of LTX-315 to reshape the tumor microenvironment makes it potentially ideal as a combination partner for other immunotherapies, such as immune checkpoint inhibitors (anti-CTLA4/anti-PD-1).

\section{References}

Papers of special note have been highlighted as: • of interest;

•• of considerable interest

1 Zasloff M. Antimicrobial peptides of multicellular organisms. Nature 415(6870), 389-395 (2002).

- A seminal review on antimicrobial peptides.

2 Ganz T. The role of antimicrobial peptides in innate immunity. Integr. Comp. Biol. 43(2), 300-304 (2003).

3 Wang G, Li X, Wang Z. APD3: the antimicrobial peptide database as a tool for research and education. Nucleic Acids Res. 44, D1087-D1093 (2016)

4 Antimicrobial Peptide Database. http://aps.unmc.edu/AP

5 Giuliani A, Pirri G, Nicoletto SF. Antimicrobial peptides: an overview of a promising class of therapeutics. Cent. Eur. J. Biol. 2(1), 1-33 (2007).

6 Brogden KA. Antimicrobial peptides: pore formers or metabolic inhibitors in bacteria? Nat. Rev. Micro. 3(3), 238-250 (2005).

7 Riedl S, Zweytick D, Lohner K. Membrane-active host defense peptides - challenges and perspectives for the development of novel anticancer drugs. Chem. Phys. Lipids 164(8), 766-781 (2011).

8 Gaspar D, SaloméVeiga A, Castanho MaRB. From antimicrobial to anticancer peptides. A review. Front. Microbiol. 4(OCT), 1-16 (2013).

- Focuses on the development of anticancer drugs from antimicrobial peptides and summarizes the different candidate peptides.

9 Rapaport D, Shai Y. Interaction of fluorescently labeled pardaxin and its analogues with lipid bilayers. J. Biol. Chem. 266(35), 23769-23775 (1991).

10 Ludtke SJ, He K, Heller WT, Harroun TA, Yang L, Huang HW. Membrane pores induced by magainin. Biochemistry 35(43), 13723-13728 (1996).

11 Gazit E, Miller IR, Biggin PC, Sansom MSP, Shai Y. Structure and orientation of the mammalian antibacterial peptide cecropin P1 within phospholipid membranes. J. Mol. Biol. 258(5), 860-870 (1996).

12 Miteva M, Andersson M, Karshikoff A, Otting G. Molecular electroporation: a unifying concept for the description of membrane pore formation by antibacterial peptides, exemplified with NK-lysin. FEBS Lett. 462(1-2), 155-158 (1999).

13 Pokorny A, Birkbeck TH, Almeida PFF. Mechanism and kinetics of $\delta$-lysin interaction with phospholipid vesicles. Biochemistry 41(36), 11044-11056 (2002).

14 Wimley WC. Describing the mechanism of antimicrobial peptide action with the interfacial activity model. ACS Chem. Biol. 5(10), 905-917 (2010).

15 Wimley WC, Hristova K. Antimicrobial peptides: successes, challenges and unanswered questions. J. Membrane Biol. 239(1-2), 27-34 (2011).

16 Schibli DJ, Nguyen LT, Kernaghan SD, Rekdal Ø, Vogel HJ Structure-function analysis of tritrpticin analogs: potential relationships between antimicrobial activities, model membrane interactions, and their micelle-bound NMR structures. Biophys. J. 91(12), 4413-4426 (2006).

17 Rekdal Ø, Haug BE, Kalaaji M et al. Relative spatial positions of tryptophan and cationic residues in helical membrane-active peptides determine their cytotoxicity. J. Biol. Chem. 287(1), 233-244 (2012).

18 Hancock REW, Haney EF, Gill EE. The immunology of host defence peptides: beyond antimicrobial activity. Nat. Rev. Immunol. 16(5), 321-334 (2016).

- Focuses on the immunomodulatory effects of host defense peptides from a systems biology perspective.

19 Bellamy W, Takase M, Yamauchi K, Wakabayashi H, Kawase K, Tomita M. Identification of the bactericidal domain of lactoferrin. Biochim. Biophys. Acta, Protein Struct. Mol. Enzymol. 1121(1-2), 130-136 (1992).

20 Gifford JL, Hunter HN, Vogel HJ. Lactoferricin. Cell. Mol. Life Sci. 62(22), 2588-2598 (2005).

21 Eliassen LT, Berge G, Sveinbjornsson B, Svendsen JS, Vorland LH, Rekdal O. Evidence for a direct antitumor mechanism of action of bovine lactoferricin. Anticancer Res. 22(5), 2703-2710 (2002).

- Reports the first study on the mechanism for the antitumour activity of bovine lactoferricin.

22 Eliassen LT, Berge G, Leknessund A et al. The antimicrobial peptide, lactoferricin $B$, is cytotoxic to neuroblastoma cells in vitro and inhibits xenograft growth in vivo. Int. J. Cancer 119(3), 493-500 (2006). 
23 Tomita M, Bellamy W, Takase M, Yamauchi K, Wakabayashi H, Kawase K. Potent antibacterial peptides generated by pepsin digestion of bovine lactoferrin. J. Dairy Sci. 74(12), 4137-4142 (1991).

24 Moore SA, Anderson BF, Groom CR, Haridas M, Baker EN. Three-dimensional structure of diferric bovine lactoferrin at 2.8 Å resolution1. J. Mol. Biol. 274(2), 222-236 (1997).

25 Hwang PM, Zhou N, Shan X, Arrowsmith CH, Vogel HJ. Three-dimensional solution structure of lactoferricin B, an antimicrobial peptide derived from bovine lactoferrin. Biochemistry 37(12), 4288-4298 (1998).

26 Eliassen LT, Haug BE, Berge G, Rekdal Ø. Enhanced antitumor activity of 15 -residue bovine lactoferricin derivatives containing bulky aromatic amino acids and lipophilic N-terminal modifications. J. Pept. Sci. 9(8), 510-517 (2003).

27 Haug BE, Strøm MB, Svendsen JSM. The medicinal chemistry of short lactoferricin-based antibacterial peptides. Curr. Med. Chem. 14(1), 1-18 (2007).

28 Yang N, Rekdal Ø, Stensen W, Svendsen JS. Enhanced antitumor activity and selectivity of lactoferrin-derived peptides. J. Pept. Res. 60(4), 187-197 (2002).

29 Haug BE, Camilio KA, Eliassen LT et al. Discovery of a 9-mer cationic peptide (LTX-315) as a potential first in class oncolytic peptide. J. Med. Chem. 59(7), 2918-2927 (2016).

- Describes the discovery of LTX-315 and the structureactivity relationships of a series of 9-mer cationic peptides.

30 Camilio KA, Rekdal Ø, Sveinbjörnsson B. LTX-315 $\left(\right.$ Oncopore $\left.^{\mathrm{TM}}\right)$ a short synthetic anticancer peptide and novel immunotherapeutic agent. OncoImmunology 3(6), e29181-e29183 (2014).

31 Camilio KA, Berge G, Ravuri CS, Rekdal Ø, Sveinbjørnsson B. Complete regression and systemic protective immune responses obtained in B16 melanomas after treatment with LTX-315. Cancer Immunol. Immunother. 63(6), 601-613 (2014).

- $\quad$ LTX-315 induced a complete regression of B16 melanomas and systemic immune responses following intralesional administration of the peptide.

32 Eike L-M, Yang N, Rekdal Ø, Sveinbjørnsson B. The oncolytic peptide LTX-315 induces cell death and DAMP release by mitochondria distortion in human melanoma cells. Oncotarget 6(23), 34910-34923 (2015).
-. The oncolytic effect of LTX-315 involves perturbation of both cell membrane and the mitochondria with subsequent release of danger-associated molecular pattern molecules.

33 Zhou H, Forveille S, Sauvat A et al. The oncolytic peptide LTX-315 kills cancer cells through Bax/Bak-regulated mitochondrial membrane permeabilization. Oncotarget 6(29), 26599-26614 (2015).

34 Forveille S, Zhou H, Sauvat A et al. The oncolytic peptide LTX-315 triggers necrotic cell death. Cell Cycle 14(21), 3506-3512 (2015).

35 Kroemer G, Galluzzi L, Kepp O, Zitvogel L. Immunogenic cell death in cancer therapy. Annu. Rev. Immunol. 31(1), 51-72 (2013).

36 Marabelle A, Kohrt H, Caux C, Levy R. Intratumoral immunization: a new paradigm for cancer therapy. Clin. Cancer Res. 20 (7), 1747-1756 (2014).

37 Yamazaki T, Pitt JM, Vétizou M et al. The oncolytic peptide LTX-315 overcomes resistance of cancers to immunotherapy with CTLA4 checkpoint blockade. Cell Death Differ. 23(6), 1004-1015 (2016).

- $\quad$ LTX-315 rapidly reprograms the tumor microenvironment by decreasing local abundance of immunosuppressive Tregs and by increasing the frequency of cytotoxic $\mathrm{T}$ cells.

38 Zhou H, Forveille S, Sauvat A et al. The oncolytic peptide LTX-315 triggers immunogenic cell death. Cell Death Dis. 7 , e2134 (2016).

39 Sharma P, Allison JP. The future of immune checkpoint therapy. Science 348(6230), 56 (2015).

40 Camilio KA, Wang MY, Nestvold J, Mælandsmo G, Sveinbjørnsson B, Rekdal Ø. The oncoloytic peptide LTX315 enhances $T$ cell clonality and induces synergy with chemotherapy. Presented at: The 108th Annual Meeting of the American Association for Cancer Research. Washington, DC, USA, 1-5 April 2017.

41 Spicer J, Awada A, Brunsvig P et al. Intra-tumoral treatment with LTX-315, an oncolytic peptide immunotherapy, in patients with advanced metastatic disease induces infiltration of CD8 effector T-cells and regression in some injected tumors. Presented at: The 8 th European Cancer Congress (ECC2015). Vienna, Austria, 26 September 2015. 